\title{
Identificação dos requisitos necessários para um checklist de cirurgia cardíaca
}

\section{segura}

\author{
Identifying the necessary requirements for a safe cardiac surgery checklist \\ Identificación de los requisitos necesarios para una lista de verificación de cirugía cardíaca segura
}

Recebido: 18/10/2021 | Revisado: 29/10/2021 | Aceito: 06/11/2021 | Publicado: 11/11/2021

\author{
Thaís Honório Lins Bernardo \\ ORCID: https://orcid.org/0000-0002-8058-8400 \\ Universidade Federal de Alagoas, Brasil \\ E-mail: thais.bernardo@eenf.ufal.br \\ Iasmin Maria Ferreira da Silva \\ ORCID: https://orcid.org/0000-0002-8781-2241 \\ Universidade Federal de Alagoas, Brasil \\ E-mail: iasminferreira.ms@gmail.com \\ Sanayara Tavares Lima \\ ORCID: https://orcid.org/0000-0002-0478-6018 \\ Universidade Federal de Alagoas, Brasil \\ E-mail: sanayaratavares@hotmail.com \\ Alba Regina Cartaxo Sampaio Thomé \\ ORCID: https://orcid.org/0000-0002-5385-1357 \\ Universidade Federal de Alagoas, Brasil \\ E-mail: regina.cartaxo@outlook.com \\ Imaculada Pereira Soares \\ ORCID: https://orcid.org/0000-0002-5583-2547 \\ Universidade Federal de Alagoas, Brasil \\ E-mail: imaculada.soares@esenfar.ufal.br \\ Rogério César Correia Bernardo \\ ORCID: https://orcid.org/0000-0003-2697-3671 \\ Universidade Federal de Alagoas, Brasil \\ E-mail: rccbernardo@eenf.ufal.br \\ Fabianny Torres de Oliveira \\ ORCID: https://orcid.org/0000-0001-6193-2002 \\ Universidade Federal de Alagoas, Brasil \\ E-mail: fabianny_torres@ hotmail.com \\ Silvana Siboney Gomes da Silveira Santos \\ ORCID: https://orcid.org/0000-0002-7044-2491 \\ Universidade Federal de Alagoas, Brasil \\ silvana.gomsantos@gmail.com
}

\begin{abstract}
Resumo
Objetivo: Identificar os requisitos necessários para um checklist de cirurgia cardíaca segura. Metodologia: Trata-se de uma pesquisa de caráter descritivo, de natureza mista, utilizando-se de levantamento de dados e entrevistas. O cenário de coleta de dados foi um hospital geral de grande porte localizado no Nordeste brasileiro que realiza cirurgias cardíacas. As entrevistas aconteceram com um questionário semiestruturado aplicado aos enfermeiros que atuavam no centro cirúrgico. O projeto foi aprovado pelo Comitê de Ética em Pesquisa sob o número CAAE 16286219.8.0000.5013 e parecer 3.606.131. Resultados: Foram realizadas cinco entrevistas, nas quais todos afirmaram que consideram importante o uso de um checklist específico para cirurgia cardíaca devido a segurança do paciente, dos profissionais e a contribuição para a gestão e a especificidade da cirurgia cardíaca. O checklist avaliado no hospital dispunha de todos os elementos listados no instrumento para observação direta e é, portanto, específico para cirurgias cardíacas. Conclusão: A partir dos resultados da pesquisa foi possível evidenciar que a percepção dos profissionais de enfermagem a respeito da importância do uso do checklist é favorável, pois mesmo que o objetivo principal seja a segurança do paciente, o checklist também é útil para gerenciar de forma mais segura o processo cirúrgico e gerar dados para construir os indicadores do hospital. $\mathrm{O}$ checklist não abrange apenas o momento da cirurgia, mas sim todo o processo cirúrgico, pois permite que a cirurgia aconteça conforme planejado e com toda segurança para o paciente.
\end{abstract}

Palavras-chave: Lista de checagem; Cirurgia torácica; Enfermagem; Segurança do paciente. 


\begin{abstract}
Objective: To identify the necessary requirements for a safe cardiac surgery checklist. Methodology: This is descriptive research of a mixed nature using data collection and interviews. The setting for data collection was a large general hospital located in northeastern Brazil that performs cardiac surgery. The interviews took place by using a semi-structured questionnaire applied to nurses who worked in the surgery room. The project was approved by the Research Ethics Committee under number CAAE 16286219.8.0000.5013 and opinion 3.606.131. Results: Five interviews were conducted. All interviewees considered important the use of a specific checklist for cardiac surgery due to the safety of the patient, professionals and the contribution to the management and specificity of cardiac surgery. The checklist evaluated in the hospital had all the elements arranged in the instrument for direct observation; therefore, being specific for cardiac surgery. Conclusion: Based on the research results, the perception of nursing professionals about the importance of using the checklist is favorable, because even if the main objective is patient safety, the checklist is also useful to manage in a safer way the surgical process and generate data to build hospital indicators. The checklist does not only cover the time of surgery, but the entire surgical process, as it allows the surgery to take place as planned and safely for the patient.
\end{abstract}

Keyword: Checklist; Thoracic surgery; Nursing; Patient safety.

\begin{abstract}
Resumen
Objetivo: Identificar los requisitos necesarios para una lista de verificación de cirugía cardíaca segura. Metodología: Se trata de una investigación descriptiva, de carácter mixto, mediante recolección de datos y entrevistas. El escenario de recolección de datos fue un gran hospital general ubicado en el noreste de Brasil que realiza cirugía cardíaca. Las entrevistas se realizaron con un cuestionario semiestructurado aplicado a enfermeras que trabajaban en quirófano. El proyecto fue aprobado por el Comité de Ética en Investigación con el número CAAE 16286219.8.0000.5013 y dictamen 3.606.131. Resultados: Se realizaron cinco entrevistas, em las cuales todos manifestaron que consideraban importante el uso de un checklist específico para cirugía cardíaca por la seguridad del paciente, de los profesionales y la contribución al manejo y especificidad de la cirugía cardíaca. El checklist evaluado en el hospital tenía todos los elementos dispuestos en el instrumento para la observación directa y es, por tanto, específico para cirugía cardíaca. Conclusión: Con base en los resultados de la investigación, se pudo evidenciar que la percepción de los profesionales de enfermería sobre la importancia de utilizar la lista de verificación es favorable, ya que si bien el objetivo principal es la seguridad del paciente, la lista de verificación también es útil para gestionar de una manera más segura. el proceso quirúrgico y generar datos para construir indicadores hospitalarios. La lista de verificación no solo cubre el tiempo de la cirugía, sino todo el proceso quirúrgico, ya que permite que la cirugía se lleve a cabo según lo planeado y de manera segura para el paciente.
\end{abstract}

Palabras clave: Lista de verificación; Cirugía torácica; Enfermería; Seguridad del paciente.

\title{
1. Introdução
}

Organizações de saúde definem o tema "Segurança do Paciente" como forma de evitar riscos e danos desnecessários ao atendimento, os quais podem levar ao comprometimento da estrutura do corpo, podendo afetar o indivíduo na sua forma física, social ou psicológica (Eskildesen et al., 2019). O centro cirúrgico é um dos ambientes com inúmeros casos de eventos adversos de hospitalização. Sua causa é multifatorial e atribuída à complexidade dos procedimentos, interação das equipes interdisciplinares e ao trabalho sob pressão, apresentando-se como o setor que está mais propenso a oferecer riscos (Thomé et al., 2017).

Diversas razões ainda são apontadas para a ocorrência de efeitos adversos na cirurgia, como a precária comunicação e a distração entre a equipe multiprofissional, a não conferência da identificação do paciente e de materiais durante a assistência, entre outros, que constituem indicadores importantes para elevar a propensão de erros (Giannattasio \& Taniguchi, 2016).

Muitos desses eventos adversos podem ser evitados, sendo assim, para auxiliar na segurança do paciente, a Organização Mundial da Saúde (OMS) lançou em 2009 a Lista de Verificação para Segurança Cirúrgica (Surgical Safety Checklist). Este checklist passou a ser utilizado em todos os procedimentos cirúrgicos, independentemente do seu grau de complexidade, de qualquer hospital do mundo, cujo objetivo é auxiliar as equipes cirúrgicas a seguir, de forma sistemática, passos críticos de segurança (OMS, 2009; Thomé et al., 2017).

A literatura assegura que na prática de saúde, listas de verificação de segurança são projetadas para identificar antecipadamente um erro potencial que resulta em danos ao doente. Elas garantem que os procedimentos são seguidos, 
eliminando a dependência sobre a memória humana e fornecendo uma estrutura padronizada para a comunicação entre os membros da equipe (The Joint Commission, 2019; Verwey \& Gopalan, 2018).

Portanto, o presente estudo contribuiu na identificação de elementos necessários para a melhoria na qualidade da assistência em saúde aos pacientes submetidos a cirurgias cardíacas, trazendo hodiernas informações acerca de elementos relevantes para sua qualidade de vida nos aspectos pré, trans e pós operatórios. Tendo como objetivo identificar os requisitos necessários para um checklist de cirurgia cardíaca segura e como pergunta norteadora: Quais os requisitos necessários para um checklist de cirurgia cardíaca segura?

\section{Metodologia}

Trata-se de uma pesquisa de caráter descritivo, de natureza mista, utilizando-se de levantamento de dados e entrevistas. Uma pesquisa de natureza mista trata-se de uma combinação de dois outros métodos de pesquisa: Qualitativo e quantitativo, e tem como principais objetivos a generalização dos resultados qualitativos e a compreensão dos resultados quantitativos (Galvão et al., 2018).

O cenário de coleta de dados foi um hospital geral de grande porte que realiza cirurgias cardíacas localizado no nordeste brasileiro. A amostra foi composta por 5 enfermeiros que atuam no centro cirúrgico que realiza cirurgias cardíacas do referido hospital. A aproximação se deu por meio de acesso ao centro cirúrgico, mediante autorização da instituição de saúde e do Comitê de Ética em Pesquisa sob número CAAE 16286219.8.0000.5013, respeitando os princípios e diretrizes da resolução 196/96 do Conselho Nacional de Pesquisa em Saúde que envolve seres humanos. O início da coleta de dados ocorreu somente após a aprovação do CEP, considerando a apresentação do estudo, a disponibilidade do setor e a viabilidade do mesmo.

O recrutamento dos enfermeiros foi realizado durante o seu expediente de serviço, em momento oportuno. Como critérios de inclusão, estabeleceu-se que o indivíduo deveria ser enfermeiro atuante nos centros cirúrgicos do hospital sede da pesquisa há pelo menos 6 meses. Como critérios de exclusão, definiu-se: enfermeiros que estejam de férias e/ou licença médica durante o período da coleta de dados.

A coleta de dados foi realizada por meio da observação direta do checklist utilizado no hospital em que ocorreu a pesquisa, para embasar esta observação, foi utilizado um instrumento que pôde analisar tal checklist, além de entrevista aos enfermeiros para analisar por meio de um questionário semiestruturado a importância do uso de um checklist específico para cirurgia cardíaca. As entrevistas foram realizadas nos horários diurnos durante a rotina de serviço do setor.

O instrumento aplicado aos enfermeiros continha questões abertas e fechadas e foi composto por três partes, a saber: 1 - Características demográficas do enfermeiro (idade, estado civil, renda familiar, tempo de formação acadêmica em enfermagem, tempo de atuação no centro cirúrgico, titulação). 2 - Dados referentes à instituição; 3 - Dados referentes à identificação dos requisitos necessários para um checklist de cirurgia cardíaca segura. A aplicação do roteiro semiestruturado contendo as perguntas discursivas permitiu ao entrevistado discorrer livremente sobre as questões apresentadas, assim como possibilitou o aprofundamento de algumas delas pelo entrevistador.

As variáveis do estudo relacionadas aos enfermeiros foram: idade, estado civil, renda familiar, tempo de formação acadêmica em enfermagem, tempo de atuação no centro cirúrgico e titulação. As variáveis relacionadas a instituição foram: quantidade de cirurgias, tipos de cirurgias e dados referentes ao checklist como do paciente e do procedimento cirúrgico propriamente dito.

Respeitando-se a Resolução nº66, de 12 de dezembro de 2012 fez-se necessário e obrigatório a existência do Termo de Consentimento Livre e Esclarecido, respeitando a dignidade, sigilo e consentimento do entrevistado. Os dados foram analisados por meio de estatística descritiva. Nas questões abertas, as respostas foram agrupadas por semelhança, culminando em categorias. 


\section{Resultados}

Inicialmente, foi realizada a revisão integrativa, levantando dados com a finalidade de conhecer os instrumentos utilizados em cirurgias cardíacas para embasar a observação direta do checklist utilizado na instituição. A pesquisa indicou que este tipo de procedimento cirúrgico é complexo e possui especificidades, culminando nos seguintes itens: identificadores do paciente; local da cirurgia a ser operado; procedimento a ser realizado; termo de consentimento; confirmação de presença de prótese na sala de operação (SO); conferência de contagem de instrumental cirúrgico pré-operatória; risco de via aérea difícil; risco de sangramento; risco de alergia; duração do procedimento; correta esterilização dos materiais cirúrgicos; temperatura da circulação extracorpórea; conferência de contagem de instrumental cirúrgico pós-operatória e antibiótico profilático.

A pesquisa foi realizada em um hospital privado no nordeste brasileiro composto por 80 leitos e que dispõe de centro cirúrgico. Foram realizadas cinco entrevistas com enfermeiros do referido hospital. Destes 5, um é enfermeiro gerencial do setor, 2 são assistenciais exclusivos do centro cirúrgico e 2 também prestam serviço na Unidade de Terapia Intensiva (UTI). A caracterização da amostra é demonstrada na Tabela 1.

Tabela 1 - Frequência de enfermeiros entrevistados por sexo, estado civil, renda mensal e faixa etária, Brasil - 2020.

\begin{tabular}{|c|c|c|}
\hline Sexo & $\mathbf{N}$ & $\%$ \\
\hline Feminino & 4 & 80 \\
\hline Masculino & 1 & 20 \\
\hline Total & 5 & 100 \\
\hline Estado civil & $\mathbf{N}$ & $\%$ \\
\hline Solteiro & 1 & 20 \\
\hline Casado & 4 & 80 \\
\hline Divorciado & 0 & 0 \\
\hline Total & 5 & 100 \\
\hline Renda mensal & $\mathbf{N}$ & $\%$ \\
\hline De 3 a 6 salários mínimos & 3 & 60 \\
\hline De 6 a 9 salários mínimos & 1 & 20 \\
\hline Mais de 9 salários mínimos & 1 & 20 \\
\hline Total & 5 & 100 \\
\hline Faixa etária & $\mathbf{N}$ & $\%$ \\
\hline 25 a 29 anos & 2 & 40 \\
\hline 30 a 34 anos & 2 & 40 \\
\hline 35 a 39 anos & 0 & 0 \\
\hline 40 a 44 anos & 1 & 20 \\
\hline Total & 5 & 100 \\
\hline
\end{tabular}

Fonte: Dados da pesquisa (2020).

As entrevistas realizadas apontam que a amostra teve em sua maioria indivíduos do sexo feminino (80\%), casados (80\%), com renda média mensal de 3-6 salários mínimos (60\%) e que se dividem na faixa etária de 25-29 anos (40\%), 30-34 $\operatorname{anos}(40 \%)$ e $40-44 \operatorname{anos}(20 \%)$.

Em relação ao tempo de formação acadêmica/atuação em centro cirúrgico (CC) e titulação, os resultados expuseram que o tempo de formação dos enfermeiros entrevistados varia, sendo o menor de 2 e o maior de 15 anos; assim como o tempo 
médio de atuação em CC, sendo o menor 2 e o maior 10 anos. Em relação ao período de atuação no CC do hospital pesquisado, a maioria (80\%) possui experiência em outras instituições, enquanto $20 \%$ iniciou o trabalho no CC neste hospital. Todos os entrevistados possuem alguma especialização, porém somente em $20 \%$ destes ela é específica para cardiologia/hemodinâmica. Os demais são especialistas em urgência e emergência (60\%), terapia intensiva (80\%), dermatologia (20\%) e nefrologia (20\%), como expõe a Tabela 2.

Tabela 2 - Dados referentes ao coordenador da lista de verificação, Brasil - 2020.

\begin{tabular}{ccc}
\hline Utilização de checklist de cirurgia cardíaca & N & $\%$ \\
\hline Sim & 5 & 100 \\
Não & 0 & 0 \\
Total & 5 & 100 \\
\hline Profissionais que aplicam o checklist na SO & N & $\%$ \\
\hline Enfermeiro & 4 & 80 \\
Cirurgião & 1 & 20 \\
Anestesista & 1 & 20 \\
Técnico em Enfermagem & 5 & 100 \\
Auxiliar de Enfermagem & 0 & 0 \\
Residente & 1 & 20 \\
Total & 5 & 100 \\
\hline
\end{tabular}

Fonte: Dados da pesquisa (2020).

Durante a coleta de dados, identificou-se que todos os enfermeiros entrevistados informaram que a instituição de saúde utiliza checklist de cirurgia cardíaca segura. Quanto ao questionamento sobre quais profissionais aplicam o checklist na sala de operação (SO), 100\% incluiu o técnico de enfermagem em suas respostas, $80 \%$ incluiu a/o enfermeira(o) e $20 \%$ incluiu o cirurgião, anestesista e residente de cirurgia cardíaca/anestesia.

Nas questões abertas, as respostas dos entrevistados acerca da importância do uso de um checklist específico de cirurgia cardíaca foram agrupadas por semelhança, culminando nas categorias: segurança do paciente; segurança dos profissionais; organização da gestão hospitalar e complexidade da cirurgia cardíaca, exposto na Tabela 3. Ademais, todos afirmaram que consideram importante o uso de um checklist específico para cirurgia cardíaca e os mesmos relataram:

"Sim. É importante porque é uma forma de gerenciar de forma mais eficaz e segura todo o processo cirúrgico, além de facilitar o trabalho de forma geral, garantindo mais controle e segurança até mesmo para a gestão e infraestrutura" (Enfermeiro 1).

"Sim, assegura maior evidência na cirurgia segura voltada para cirurgia cardíaca, pois este tipo cirúrgico envolve fatores específicos direcionados a profissionais específicos com riscos associados a cada profissional" (Enfermeiro 2 - gerencial).

"Sim. É com o checklist que se torna possível avaliar toda a sistemática da cirurgia, principalmente na cardíaca, que é de alta complexidade e possui muitas especificidades como válvulas, coronária, tipo sanguíneo, pressão arterial média, que precisam estar checadas. $O$ checklist também ajuda na gestão, pois gera dados para construir os indicadores do hospital, como quantidade de pinças cirúrgicas do hospital, avaliação de risco de queda, flebite, lesão por pressão, lateralidade, broncoaspiração, além de diminuir bastante o risco para o paciente, que é o ator principal, e hoje com a segurança do paciente, ele tem que estar 100\% seguro, pois entrega a vida a nós" (Enfermeiro 3). 
"Sim. É importante para a segurança dos processos e do paciente, além de fornecer dados para indicadores assistenciais. $O$ checklist de cirurgia cardíaca engloba assistência não só da enfermagem, mas do cirurgião, anestesista, pois ele inicia-se desde a marcação da cirurgia, caso haja necessidade de materiais específicos ou diferentes, tem-se por obrigação avisar com antecedência, para que não haja correria no momento da cirurgia, então contribui também para a organização. O checklist não engloba só o momento, mas sim todo o processo cirúrgico" (Enfermeiro 4).

"Importantíssimo para que todo processo cirúrgico aconteça conforme planejado e com toda segurança para o paciente, reduzindo praticamente $100 \%$ os riscos de danos aos pacientes" (Enfermeiro 5).

Tabela 3 - Dados referentes às falas dos entrevistados, Brasil - 2020.

\begin{tabular}{ccc}
\hline $\begin{array}{c}\text { Elementos citados sobre a importância do uso do checklist } \\
\text { específico de cirurgia cardíaca }\end{array}$ & N & $\%$ \\
\hline Segurança do paciente & 5 & 100 \\
Segurança dos profissionais & 5 & 100 \\
Organização da gestão hospitalar & 3 & 60 \\
Complexidade da cirurgia cardíaca & 3 & 60 \\
Total & 5 & 100 \\
\hline
\end{tabular}

Fonte: Dados da pesquisa (2020).

Observa-se que todos os enfermeiros citaram a segurança do paciente e dos profissionais como elementos importantes no uso correto do checklist de cirurgia cardíaca, enfatizando ainda mais o quão necessário é este instrumento no ambiente cirúrgico. Destaca-se, ainda, a organização da gestão hospitalar e a complexidade da cirurgia cardíaca, ambas citadas por $60 \%$ dos entrevistados, evidenciando que o seu uso correto proporciona benefícios desde a gestão até a assistência.

Com a observação direta, observou-se que o checklist avaliado no hospital dispunha de todos os elementos dispostos no instrumento, a saber: Identificadores do paciente; local da cirurgia a ser operado; procedimento a ser realizado; termo de consentimento; confirmação de presença de prótese na SO; conferência de contagem de instrumental cirúrgico pré-operatória; risco de via aérea difícil; risco de sangramento; risco de alergia; duração do procedimento; correta esterilização dos materiais cirúrgicos; temperatura da circulação extracorpórea; conferência de contagem de instrumental cirúrgico pós-operatória e antibiótico profilático.

\section{Discussão}

Durante as entrevistas, evidenciou-se que todos os participantes do estudo afirmaram considerar importante o uso do checklist de cirurgia segura, corroborando com a literatura, que assegura que na prática de saúde, listas de verificação de segurança são projetadas para identificar antecipadamente um erro potencial que resulta em danos ao paciente. Elas garantem que os procedimentos são seguidos, eliminando a dependência sobre a memória humana e fornecendo uma estrutura padronizada para a comunicação entre os membros da equipe (The Join Commission, 2019, Verwey \& Gopalan, 2018).

Quando se trata das circunstâncias desta pesquisa, é importante ressaltar que as doenças cardiovasculares lideram as causas de morte no Brasil, bem como no mundo, exigindo constantemente novas estratégias para o seu contorno; os procedimentos cirúrgicos, por sua vez, são cada vez mais frequentes na assistência, sendo a cirurgia cardíaca considerada de grande porte e de alta complexidade, corroborando com a fala de alguns enfermeiros entrevistados. Além disso, por ser um procedimento complexo, a cirurgia cardíaca requer um tratamento adequado, específico e individualizado em todas as suas fases operatórias (Pereira et al., 2016; Reis et al., 2019; Taurino, 2019). Nesse sentido, implementar práticas que favoreçam a saúde do paciente e acrescentem segurança ao processo deve ser sempre incentivada. 
A aplicação completa da lista de verificação da cirurgia segura é fundamental para reduzir os danos ao paciente cirúrgico. Protocolos internos com adaptações do checklist às especificidades e complexidade da prática assistencial da instituição são recomendados, assim como ocorre na instituição deste estudo, que possui o checklist adaptado para cirurgias cardíacas, elevando sua funcionalidade e assertividade. É importante que o uso deste instrumento seja frequentemente monitorado utilizando um plano de melhoria contínua (Gutierres et al., 2018).

A criação da Lista de Verificação de Segurança Cirúrgica teve o intuito de proporcionar uma melhoria na qualidade da assistência à saúde, contribuindo para garantir maior segurança durante os procedimentos cirúrgicos, proporcionando a diminuição da incidência de erros preveníveis, possibilitando diálogo e atividade em equipe no âmbito da saúde e, consequentemente, fortalecendo ações seguras; portanto, é essencial que os profissionais compreendam a importância de uma cirurgia segura, e não a enxerguem somente como uma imposição organizacional (Borba et al., 2020; Gehres, Cremonese \& Schuh, 2020). Durante a coleta de dados desta pesquisa, foi possível perceber que os profissionais dispunham de conhecimento acerca da relação entre importância da cirurgia segura e práticas como a utilização do checklist.

Em todas as entrevistas acerca da importância da utilização do checklist, foi citada a segurança do paciente, fator primordial dentro de uma instituição de saúde e que exige um trabalho em equipe feito com eficiência e clareza, sendo a troca de conhecimentos fator que favorece sua melhoria. Ademais, a qualidade do cuidado em saúde está diretamente relacionada à segurança do paciente e no ambiente do centro cirúrgico, que é bastante técnico e específico, ela assume uma importância ainda maior, portanto, instrumentos e atitudes que proporcionem a segurança do paciente e dos profissionais devem ser aderidos e implementados, como o uso do checklist de cirurgia segura da OMS (Ribeiro et al., 2017; Silva et al., 2021).

No contexto da sala de cirurgia, a busca pela segurança e qualidade do atendimento no período intraoperatório configurou-se como uma importante atividade gerencial do enfermeiro. A enfermagem está presente em todas as etapas do período perioperatório, sendo considerada a principal equipe e agente de mudança para a transformação do sistema de saúde, a fim de torná-lo mais seguro. No ambiente cirúrgico, o enfermeiro tem papel fundamental na garantia de que as melhores práticas de cuidado proporcionam segurança ao paciente (Gutierres et al., 2018).

Dessa forma, fica claro que a equipe de enfermagem é peça fundamental na eficiência da aplicabilidade do checklist, e, consequentemente, se não estiver preparada ou não possuir o conhecimento acerca do instrumento e da sua importância, a avaliação fica comprometida.

Uma pesquisa cujo objetivo foi compreender o conhecimento e práticas dos técnicos de enfermagem sobre a aplicação do checklist de cirurgia segura, concluiu que esta classe não dispunha do conhecimento científico necessário acerca do assunto, encarando as medidas de segurança como normas da instituição, não compreendendo seu papel na segurança do paciente; desta maneira, ações constantes de educação permanente são essenciais nos serviços, buscando garantir a melhor assistência ao paciente, visto que os técnicos em enfermagem frequentemente são os encarregados de aplicar o checklist na sala de cirurgia. O desconhecimento da equipe de enfermagem acerca da importância da cirurgia segura e de seus instrumentos pode comprometer a assistência e é considerado como fator adverso (Gehres, Cremonese \& Schuh, 2020; Ferreira, Ribeiro, Mendonça \& Amaro, 2019).

A enfermagem é a única profissão da área da saúde em que a formação possui a teoria juntamente da prática quando se trata da gestão/administração em saúde, destacando a importância de indicadores confiáveis para o gerenciamento e a assistência de qualidade, além de possibilitar a identificação de riscos evitáveis, portanto, esses profissionais possuem potencial para contribuir na segurança do paciente, como assegura a pesquisa de Gutierres et al (2018), que descreve as recomendações de enfermeiros para boas práticas de segurança do paciente em centro cirúrgico, sendo uma delas a utilização do checklist de cirurgia segura, apontando-a como fundamental para a redução de danos ao paciente cirúrgico (Koerich, Lanzoni \& Erdmann, 2016). 
Apesar de todos os benefícios comprovados após a aplicação do checklist, existem evidências de que a sua adesão é baixa, especialmente em países em desenvolvimento, como o Brasil. Ainda há muitas barreiras a serem superadas para a implementação do instrumento, dentre elas, a resistência dos próprios profissionais de saúde, a falta de treinamento pela instituição e de tempo para que a equipe se dedique ao protocolo, a deficiência de infraestrutura, predominando, no entanto, na maioria dos países a falha na comunicação entre a equipe multiprofissional, fator que deveria ser melhorado com a própria aplicação do checklist. A comunicação efetiva, bem como o trabalho em equipe, são capazes de proporcionar uma assistência segura e de qualidade ao paciente e seus familiares, sendo um dos principais causadores de eventos adversos as falhas em sua execução; no entanto, para que a comunicação flua de forma eficaz, a equipe deve possuir relações de confiança entre si e com a gestão (Arboit et al., 2020; Madalozzo et al., 2021; Oliveira, Abreu \& Almeida, 2017; Rocha et al., 2020).

Ademais, existem aspectos multidimensionais quando se trata da cultura de segurança do paciente; portanto, o trabalho em equipe, as condições de trabalho satisfatórias, a comunicação efetiva e o suporte de liderança são fatores que favorecem que tal cultura seja bem desenvolvida, contribuindo para a adoção de medidas preventivas e aprendizado organizacional, por exemplo; sendo assim, o trabalho multiprofissional é primordial para que seja oferecido ao paciente uma assistência integral e segura.

\section{Conclusão}

Por meio deste estudo, observou-se que na instituição onde a pesquisa foi feita, a aplicação do checklist é realizada majoritariamente pelos profissionais de enfermagem. Com isso, nota-se que apesar de apenas $20 \%$ dos enfermeiros entrevistados possuírem especialização em cardiologia/hemodinâmica, a maioria da equipe de enfermagem desse hospital foi capacitada e devidamente instruída para realizar essa aplicação.

A partir dos resultados da pesquisa foi possível evidenciar que a percepção dos profissionais de enfermagem a respeito da importância do uso do checklist é favorável, pois mesmo que o objetivo principal seja a segurança do paciente, o checklist também é útil para gerenciar de forma mais segura o processo cirúrgico e gerar dados para construir os indicadores do hospital. O checklist não abrange apenas o momento da cirurgia, mas sim todo o processo cirúrgico, pois permite que a cirurgia aconteça conforme planejado e com toda segurança para o paciente.

É primordial para a segurança do paciente e da equipe que este protocolo seja instituído e incorporado no dia a dia de mais centros cirúrgicos, sendo capaz de evitar danos e prejuízos à saúde da pessoa que está sendo submetida a um procedimento cirúrgico, além de garantir mais autonomia e proteção ao profissional e à instituição como um todo.

\section{Referências}

Arboit, E. L., Bellini, G., Schutz, C. R., Moraes, M. C. S., aKinalski, S. S. \& Barcellos, R. A. (2020). A cultura de segurança do paciente na perspectiva multiprofissional. Research, Society and Development, v. 9, n.5, e125953088.

Borba, G. M. N., Queiroz, V. C., Lins, M. L. V., Alves, S. R. P., Silva, P. E. \& Souza, A. P. M. A. (2020). Cirurgia segura: a importância do checklist na visão do enfermeiro. International Journal of Development Research, 10, (08), 39373-39378.

Eskildesen, L., Forti, A., Paião, L. \& Magri, M. A. (2019). Aplicação da escala ELPO em pacientes submetidos à cirurgias cardiovasculares. Cuid Enferm. 13(2):116-121.

Ferreira, N. C. S., Ribeiro, L., Mendonça, E. T. \& Amaro, M. O. F. (2019). Checklist de Cirurgia Segura: Conhecimento e Utilização do nstrumento na Perspectiva dos Técnicos de Enfermagem. Revista de Enfermagem do Centro Oeste Mineiro. 9/2608.

Galvao, M. C. B., Pluye, P., \& Ricarte, I. L. M. (2017). Métodos de pesquisa mistos e revisões de literatura mistas: conceitos, construção e critérios de avaliação. InCID: Revista De Ciência Da Informação E Documentação, 8(2), 4-24.

Gehres, A. R. D., Cremonese, L. \& Schuh, L. X. (2020). Implantação e adesão do checklist de cirurgia segura em instituições hospitalares: revisão integrativa. Research, Society and Development, v. 9, n.8, e 97985093. 
Research, Society and Development, v. 10, n. 14, e491101421968, 2021

(CC BY 4.0) | ISSN 2525-3409 | DOI: http://dx.doi.org/10.33448/rsd-v10i14.21968

Gutierres, L. S., Santos, J. L. G., Peiter, C. C., Menegon, F. H. A., Sebold, L. F. \& Erdmann, A. L. (2018). Boas práticas para a segurança do paciente na sala de cirurgia: recomendações de enfermeiros. Rev. Bras. Enferm. vol.71 supl.6 Brasília.

Madalozzo, M. M., Lucas, J. I. P., Kanan, L. A., Marcon, S. R. A., Souza, A. S., Michelin, F. T., Hoffman, S. P. (2021). Cultura de segurança do paciente em um hospital acreditado de alta complexidade. Research, Society and Development, v. 10, n.6, e55510616113.

Koerich, C., Lanzoni, G. M. M. \& Ferdmann, A. L. (2016). Fatores associados à mortalidade de pacientes submetidos à cirurgia de revascularização do miocárdio. Rev. Latino-Am. Enfermagem. 24: e 2748.

Oliveira, A. C., Abreu, A. R. \& Almeida, S. S. (2017). Implementação do checklist de cirurgia segura em um hospital universitário. Enferm. foco 8 (4): 14-18.

Organização Mundial da Saúde. (2009). Segundo desafio global para a segurança do paciente: Cirurgias seguras salvam vidas. Rio de Janeiro: Organização Pan-Americana da Saúde/Ministério da Saúde/Agência Nacional de Vigilância Sanitária.

Pereira, K. S. M., Oliveira, J. C. P., Carvalho, F. C. \& Bellen, B. (2016). Complicações cardíacas em cirurgia vascular. J. vasc. bras., Porto Alegre, v. 15, n. 1, p. $16-20$.

Reis, M. M. R., Lima, E. F. A., Casagrande, R. I., Fioresi, M., Leite, F. M. C. \& Primo, C. C. (2019). Perfil epidemiológico de pacientes submetidos à cirurgia cardíaca. Rev enferm UFPE on line., Recife, 13(4):1015-22.

Ribeiro, H. C. T. C., Quites, H. F. O., Bredes, A. C., Sousa, K. A. S. \& Alves, M. (2017). Adesão ao preenchimento do checklist de segurança cirúrgica. Cad. Saúde Pública; 33(10):e00046216.

Rocha, R.G.; Batista, D.B.S.; Pereira, E.R.; Almeida, L.F.; Fassarella, C.S.; Tavares, J.M.A.B.; Broca, P.V. (2020). Limitações na implementação da lista de checagem de cirurgia e impactos na segurança do paciente cirúrgico. Research, Society and Development, v. 9, n.9, e169997089.

Silva, B.J.R., Santos, B. D. V., Andrade, C. R., Macedo, E. R., Andrade, H. S. (2021). Ações de Enfermagem que promovem a segurança do paciente no âmbito hospitalar. Research, Society and Development, v. 10, n. 5, e44110515202.

The joint commission. (2019). National Patient Safety Goals. Recuperado de: http://www.jointcommission.org/assets/1/6/NPSG_Chapter_Jan2012_HAP.pdf.

Thomé, A. R. C. S., Melo, E. S., Silva, V. M. S., Almeida, T. G., Farias, I. P. \& Vasconcelos, E. L. (2017). Construção e validação de instrumento para assistência em cirurgia cardíaca segura. Revista de enfermagem UFPE on line, [s. l.].

Taurino, I. J. M. (2019). Cirurgia cardíaca: refletindo sobre o cuidado de enfermagem no período pós-operatório. PubSaúde, 2 , a014.

Verwey, S. \& Gopalan, P. D. (2018). An investigation of barriers to the use of the World Health Organization Surgical Safety Checklist in theatres. South African Medical Journal, [S.1.], v. 108, n. 4, p. 336-341. 